\title{
Development, Applications, Benefits, Challenges and Limitations of the New Genome Engineering Technique. An Update Study
}

Andrei Crauciuc ${ }^{1}$, Florin Tripon ${ }^{*}$, Andreea Gheorghiu ${ }^{1}$, Georgiana Nemes ${ }^{1}$, Alina Boglis ${ }^{1}$, Claudia Banescu1,2

University of Medicine and Pharmacy Tirgu Mures, Romania

2 Center for Advanced Medical and Pharmaceutical Research, University of Medicine and Pharmacy Tirgu Mureș, Romania

We assume that the CRISPR Cas9 theory must be delimited by applicability, because the consequences of long term DNA manipulation remain unknown. Moreover, the irreversibility of this procedure should instigate researchers to reserved opinions.

Usefulness as well as benefits of CRISPR Cas9 made it one of the most popular and used genome editing technique. But with its huge potential, ethical and safety concerns emerge. Therefore, before continuing research in this direction we should have a well organized system that is able to make that differentiation between research and reproduction. However we truly believe in the future of genetic engineering and with the CRISPR-Cas9 system we expect that the opportunity of treating now so called incurable diseases arises. Time is all we need.

Keywords: CRISPR-Cas9, Cpf1, Class 2 CRISPR effector, RNA guided

Received 7 November 2016 / Accepted 3 February 2017

\section{The development of genome engineering Genome engineering}

An important step for genomic engineering was achieved in 1990 by C. Srinivasana a chemist at Johns Hopkins University, who tried to manipulate bacterial enzymes which could cut DNA [1].

The first step in the genetic engineering process was the division of the chromosomal DNA using genome editing techniques such as zinc finger nucleases (ZFNs) and transcription activator-like effector nucleases (TALENs). The second step was to increase its specificity by using clustered regularly interspaced short palindromic repeats (CRISPR) associated with the Cas9 protein system (CRISPR-Cas9) which has RNA-guided (g-RNA) nucleases that precisely interrupts targeted sequences at the desired locus in the genome of any living organisms [2]. Moreover, there is a possibility to insert specific sequences into the DNA.

Genome editing tools play an important role in genetic engineering, and that is the reason why the CRISPR-Cas9 system has screen platforms which can be operated in various formats, such as the knockout, knockdown and activation screens. Their purpose is to enable high-throughput interrogation of gene functions in healthy and compromised organisms, in order to target coding and non-coding regions throughout the genome, and last but not least, to induce less off-target effects [3].

\section{The CRISPR-Cas9 system}

The CRISPR system was used for the first time in 2002 and the theory sustaining it was based on the adaptive im-

* Correspondence to: Florin Tripon

E-mail: tripon.florin.2010@gmail.com mune system of bacteria. Initially, the mechanism had a Cas9 enzyme which used RNA to guide itself on the complementary sequences of DNA $[4,5]$.

Several types of Cas enzymes have been developed, but the most well-known is called Cas9, originally discovered in Streptococcus pyogenes [7, 8, 9]. Three major types of the CRISPR-Cas system have been delineated, further divided into several subtypes and a few chimeric variants, [5] of which type II has been studied the most [6].

Scientists' have focused on the immune system possessed by bacteria that are constantly adapting to foreign invaders such as viruses and plasmids. Further analysis detected clustered regularly interspaced short palindromic repeats (CRISPRs), meaning that there are short repetitons separated by nonrepetitive sequences, called "spacer DNA," acquired from an early encounter with foreign DNA.

The nuclease Cas (CRISPR associated proteins) uses spacer DNA to identify and crop the DNA of the invaders. Moreover, in association with a guide RNA, Cas is directed towards a specific target which needs to be removed leading to a resistance to the attack of the virus.

This process could be applied to the nucleus of any living cell. The system would attach a DNA sequence followed by the Cas9 which would unzip the genome, pair it to the target RNA, and two molecules would perform the cut. Afterwards, a repairing process would be initiated, however there would be a high risk of random mutations resulting in a disabled gene. Researchers have the option to replace the mutant sequence with a healthy new version in stem cells or fertilised eggs and to target more than one gene at once, making the CRISPR Cas 9 system an im- 
portant tool for studying complex diseases and improving different biotechnologies [10,11]. Figure 1 illustrates the process of CRISPR-Cas9 associated system.

\section{New proteins discovered (class 2 CRISPR effector)}

In the last quarter of 2015, B. Zetche and F. Zhang reported another class of CRISPR effector namely Cpf1 proteins. The purpose of their discovery was to find a safer approach in this technique and to overcome its current limitations. From the total of 16 Cpf1 enzymes discovered, two are compatible with human cells. The CRISPR-Cpf1 technique has the following advantages: a Cpf1 small size enzyme, a single g-RNA molecule compared to Cas9 which needs two molecules and the DNA lysis being produced in several places offering multiple choices. Moreover, the lysis has a different approach by creating "sticky end" which is easier to control compared to Cas9 which creates "blunt ends". Another positive aspect of the "sticky end" is the fact that the insertion of DNA can be directed via the information this procedure carries, making it more manageable $[13,14,15]$. The Cpf1 enzyme is safe to use during the procedure because it is easy to handle [14]. Research institutes have recognised the importance of these new findings and have made the legal preparations in order for it to be beneficial to other researchers and to incorporate the enzyme into their CRISPR system for superior scientific findings [16]. As F. Zhang explained "We are committed to making the CRISPR-Cpf1 technology widely accessible. Our goal is to develop tools that can accelerate research and eventually lead to new therapeutic applications. We see much more to come, even beyond Cpf1 and Cas9, with other enzymes that may be repurposed for further genome editing advances $[14,17] . "$

\section{Applications and Benefits}

The CRISPR-Cas9 system is applied in various scientific fields, such as: medicine and biology, pharmacology and biotechnology engineering.

\section{Medicine}

An area where the CRISPR-Cas9 system has provided its advantages was that of genetic recessive disorders. To investigate the possibility of gene correction in adult stem cells using CRISPR-Cas9, the focus was on cystic fibrosis (CF), where adult intestinal stem cells were isolated and expanded in culture, then the mutant F508del allele was corrected using the CRISPR-Cas9 and the functionality of the targeted allele was demonstrated $[18,19]$.

Another application of this system is in sickle-cell anaemia and Duchene muscular dystrophy. Additionally, Cas9 can be used to correct the causative mutation in dominant negative disorders such as transthyretin-related hereditary amyloidosis or different forms of retinitis pigmentosa [20].

Huntington's is an autosomal dominant neurodegenerative disease caused by trinucleotide repetition (CAG) in the HTT gene after 40 CAG repeats, the majority of patients develop the signs and symptoms of the disease $[21,22]$. The CRISPR-Cas9 system could treat this illness by excluding the trinucleotide repetition from the patient's DNA. By bringing together the CRISPR-Cas9 system and induced pluripotent stem cell (IPSC) the editing process could be improved [24], and there are several strategies for this procedure [25]. Besides Huntington's disease, this technique could be performed in Rett syndrome [26] and schizophrenia [27].

An enormous milestone is about to be attained in cancer research by providing new methods to configure can-

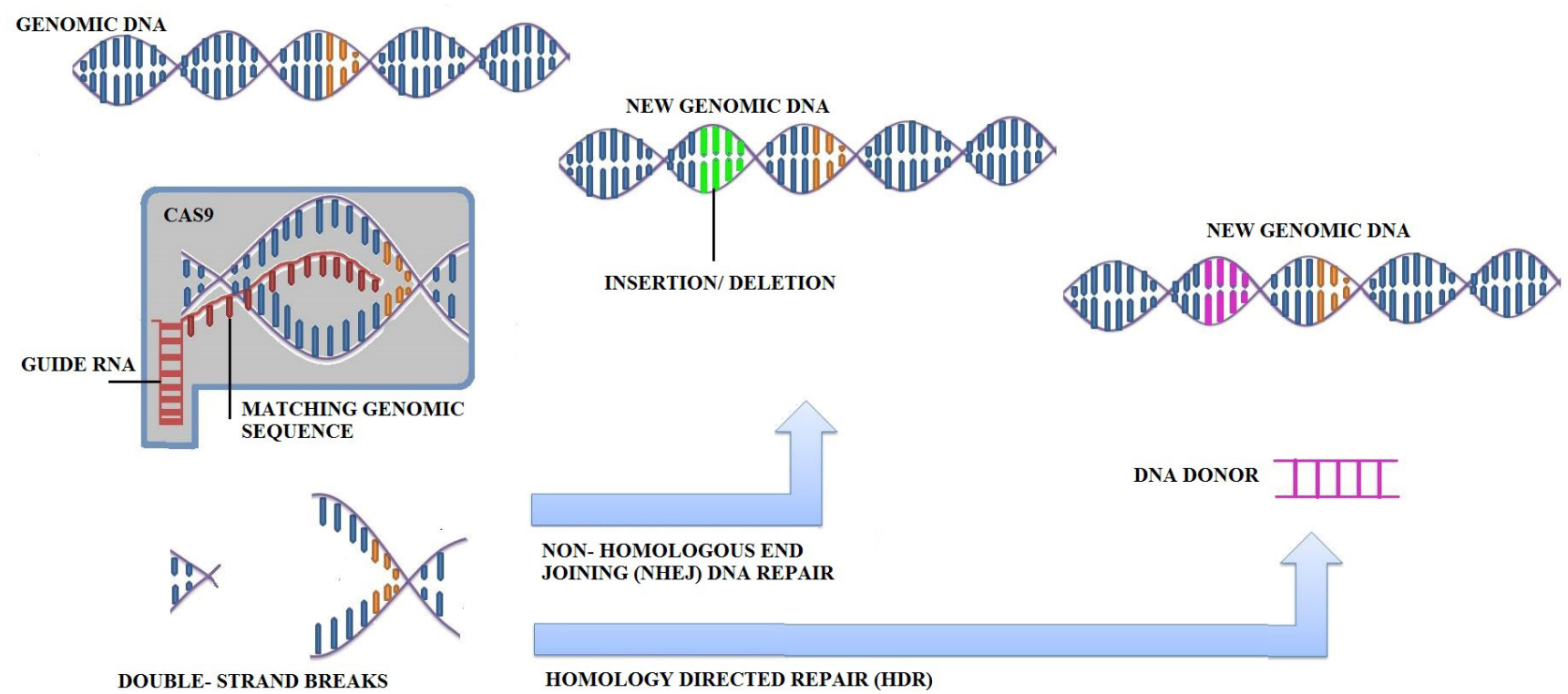

Fig. 1. The process of the CRISPR-Cas9 system. The g-RNA and Cas 9 enzyme induce the bicatenar deletion of a targeted DNA fragment, followed by DNA repair methods which either restore the DNA sequence or fail to do so. As a consequence, the genome could become unstable due to chromosomal aberrations such as translocations or deletions if the double strand breaks (DSBs) remain unrepaired or misrepaired. Apoptosis could be induced, and so could senescence, which leads to the loss of heterozygozity [12]. The NHEJ and homology directed repair (HDR), DNA repair methods for DSBs are complex and we elaborated on them in a short description here. Other molecules which take part in the process are: Ku70, Ku80, DNA PK-activation, Ligase IV, XRRC4, XLF, MRN, ATM, Rad 51. 
cer progression in vivo. The main focus is on editing and manipulating the genome of somatic cells in mammals by using basic, flexible and cost-effective elements [28]. Progressive genetic and epigenetic alterations will eventually develop cancer in a living organism and the CRISPR-Cas9 system has variable components with applications in treating and modelling this illness. Currently, there are trials focused on modelling oncogenic mutation in cell lines [29], adult animals [30] disabling oncogenic viruses [31] or manipulating the cancer genome [32].

The epigenome and transcriptome are important causes of cancer genesis, and their consistent exploitation is beneficial in improving the therapy and manipulation of cancer [33].

Concerning prostate cancer cells, a flexible aptamerliposome CRISPR-Cas9 chimera which integrated an RNA aptamer was used for specific attachment to the cells' membrane, while the liposome distributed the CRISPR/ Cas9 system that targeted the polo-like kinase 1 in the cells. The results observed in vitro showed a gene inhibition effect, and in vivo, tumor regression [34]. Moreover, the CRISPR- Cas9 editing tool revealed targets for Ibrutinib therapy intended to deactivate the pre B cell receptor signal in acute lymphoblastic leukemia, such as B lymphocyte kinase and Bruton's tyrosine kinase [35].

In addition, a previously unimaginable perspective for CRISPR-Cas9 have been reported in personalised medicine (Figure 2). Regarding lung cancer, biopsy samples were taken from patients' tumours and epidermal growth factor receptor (EGFR) mutant genes were identified. With a virusdelivered CRISPR-Cas 9 editing tool the mutation could be detached or even destroyed meaning that in the future "molecular surgery" could be performed on DNA specifically targeting the disease. Nevertheless, a combination of therapies could be used for the patients' benefit, such as: radiation and chemotherapy, and even traditional surgery [36].

\section{Biotechnology engineering and applications in Phar- macology}

In the pharmaceutical industry, the CRISPR and Cre/Lox system played an important role when the development

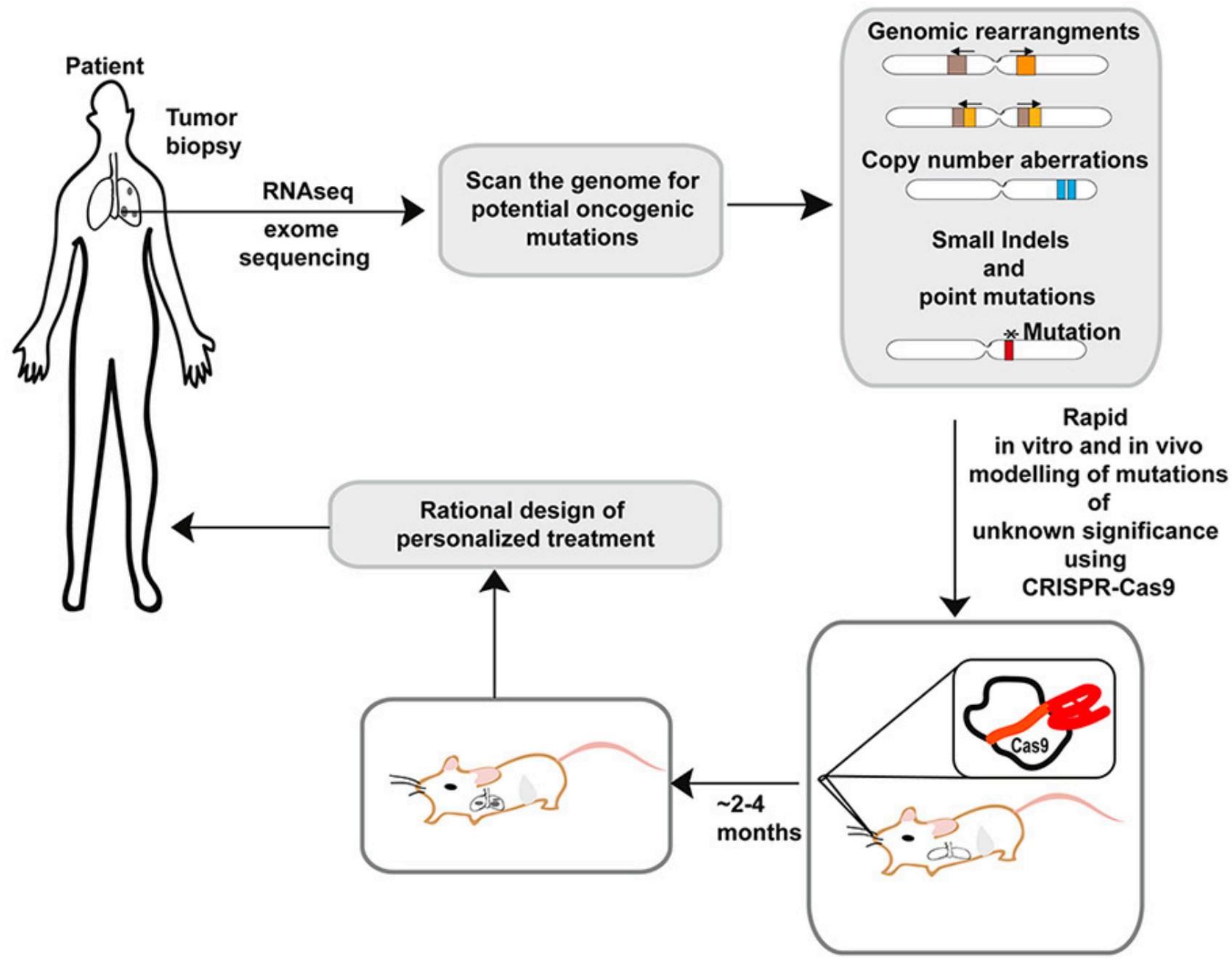

Fig. 2. Applications of CRISPR-Cas9 in personalized medicine (republished with the permissions of Andreea Ventura and Swiss Med Wkly editor-in-chief. Reference no. 28: https://smw.ch/index.php?id=75\&tx_ezmjournal_articledetail[identifier]=smw.2015.14230). The importance of this procedure in personalised medicine is the fact that it allows the discovery of functional consequences given by DNA sequences or mutations with unknown pathogenesis and repercussions. The first step consists of the discovery of these mutations in the patient's biopsied tumour tissue. The second step is the creation of these mutations in vitro and in vivo and via the documentation of the functional repercussions (in this example, the case of a mouse). Such information can be used to design an optimised personalised treatment [28]. 
of a vaccine for the Pseudorabies virus took place with the help of gene editing technology [37]. This technology provided new insights when post-vaccine immunity was inefficient and the evolution of the virus needed to be stopped.

Companies have started to invest in this revolutionary technique. There may be a financial purpose for private companies, as they have invested in the majority of the projects involving the CRISPR system.

„Interest in the genes editing forces of CRISPR-Cas9 by pharmaceutical companies literally exploded in the last year", said Bill Lundberg [38].

Their aim was to engineer immune cells and blood stem cells, discover drugs, new targets in preclinical models for a variety of disease areas and to breed plants for agricultural uses.

Nowadays there are therapies for the suppression of HIV-1 replication, yet there are none for the deactivation of the virus in the latent reservoirs. Using the CRISPRCas9 system in combination with two antiviral gRNAs the blocking of viral replication could be attained [39]. Another disease to which this system brings therapeutic value is hepatitis $\mathrm{B}$; knowing that the mini-chromosomal cccDNA (covalently closed circular DNA) generates a new viral antigen, the editing tool could be used to remove cccDNA which would lead to lower HBsAg levels [40].

There is a large number of scientific papers published revealing the successful application of the CRISPR-Cas9 technique, and we would like to mention some of these: the dicot and monocot plant genome [41], plant suspension cells with applications in pharmacology [42], bacteria, zebra fish, non-viable human embryos, mammalian cells, insect-resistant and herbicide-tolerant plant varieties, vaccine development and others. „In the short term, the development of new techniques or the improvement of existing ones will provide further instruments to counter the appearance of resistant weeds and insects and to reduce the use of agrochemicals" [43].

\section{Challenges and Limitations}

The delivery of g-RNA and the Cas9 protein has been a constant challenge, but the researchers used plasmids, viruses, and ribonucleoproteins (RNPs) as method for optimizing their gene-editing procedures, even though these three delivery methods had limitations $[44,45]$.

One of the most significant limitations of the CRISPRCas9 system was the appearance of certain off-target effects, although these represented a small percentage compared to previous techniques of genetic engineering. Various strategies had been reported to reduce off-target effects [46, $47,48]$. It is very important to select an optimizing RNA guide, Cas9 enzyme and unique target sequences [23]. Moreover, CRISPR-Cpf1 has recently shown promises in diminishing these effects. Additionally, F Zhung maintains that "Cpf1 may overcome one of CRISPR-Cas9's few limitations. The system works well for disabling genes, it is often difficult to truly edit them by replacing one DNA sequence with another" [13].
A limitation and a constant provocation at the same time was the protospacer sequence in the g-RNA represented by the 18-20-nt spacer region, and which the fundamental reason for off-target and on-target effects in CRISPR experiments [45]. Twenty DNA bases pairs (bp) are the maximum target for the CRISPR-single RNA guide ( $\mathrm{sgRNA}$ ) [23]. Thus, no more than 20bp deletions and insertions can be done.

The precision of Cas9 was found to be enormously lower than in the theoretical approach [45]. As a result, new concepts have gone into development, such as: a more accurate Cas9 system, more effective deliveries and more powerful sgRNA [49, 50].

Other researchers found a large number of unexpected mutations, described as off-target effects, which means that the CRISPR-Cas9 system interfered with other regions. Furthermore, there were excessive and unwanted errors made on mice embryos or adult human cells compared to other studies, although we must mention that they only analysed the exons. If they would have had analysed the whole genome, the number of mutations would probably have increased [50].

The consequences were not described in the literature due to the fact that the subjects and their descendants were not monitored for a long period of time. Thus, the consequences were unknown mainly because only a few studies had the objective of following the off-target effects.

Even though this is a controversial subject, there have been studies which reported a minimal percentage of inducing tumours as adverse reactions $[51,52,53]$.

Therapeutic applications have encountered challenges and limitations, since the CRISPR-Cas9 must be precisely defined or improved in the case of safe long term usage in human disease treatments [56]. Depending on DNA's complexity, CRISPR-Cas9 specificity decreases. Therefore, new challenges arise in adapting these techniques arise due to a low proportion of off-target effects seen in bacteria, zebra fish and mice compared to human cells.

This evolution in molecular genetics offers many promises, but it also brings an important ethical debate which was emphasized by use of the technique on human embryos.

The international bioethics committee of UNESCO considers that the CRISPR Cas9 system should only be used as a preventative, diagnostic and therapeutic procedure, without modifying the descendants' genome. Until now, this technique has held the possibility of treating certain diseases, such as: sickle cell anemia, cystic fibrosis and several types of cancer [57].

In March 2015, the researchers who worked to develop CRISPR acknowledged the unsafeness of this method and highlighted the topic of eugenics [49].

At the same time, using the CRISPR-Cas9 method, Chinese scientists have tried to edit the human genome by modifying the gene responsible for beta thalassemia (HBB). In order to avoid ethical concerns, they operated on nonviable embryos [50]. The technique involved the 
injection of the CRISPR-Cas9 system into 86 embryos, of which 71 survived. Of the surviving embryos, 54 were tested, only 28 embryos dividing successfully, but not all of them had the replaced genetic information. Following this experiment, the scientists ended the trial and declared that the technology was imperfect, and in order to be used on viable embryos it should have a $100 \%$ success rate [50].

They also declared that there were no reasons for ethical concerns because the experiments were made on nonviable embryos.

In December 2013, the European Union Council and Parliament adopted Horizon 2020 framework which allows funding human embryo research if all it's clauses and procedures are accomplished. Currently human gene editing is permitted in 18 Member States, whilst 3 prohibit it and the rest have no specific legislation.

Recently in Great Britain, researchers have received the approval to genetically edit viable human embryos, which are to be donated by patients taking part in in vitro fertilization procedures. The scientific study would end in 7 days, and after this time the embryos would be eradicated due to ethical concerns [55] because according to current legislation human embryos cannot be used in the blastocyst stage.

Their opponents were worried about the consequences these interventions would have on the human genome, especially in future generations. Likewise, the risk encountered in clandestine exploitation and parents being tempted with a genetically modified embryos in order to gain physical and intellectual traits for their offspring after birth [54].

\section{Conclusion}

Without any doubt, the CRISPR system holds many perspectives in the near future, leading to new enzymes being discovered and used as editing tools. For instance, Cpf1 enzyme can be easily and precisely manipulated compared to Cas9 with the purpose of obtaining aimed results without off-target effects. Despite the greater benefits and potential of this technique there is the need for well defined rules and legislations for a consistent worldwide implementation.

\section{Conflict of interest}

None to declare.

\section{References}

1. Yang GK, Jooyen C, Srinivasan C. Hybrid restriction enzymes: Zinc finger fusions to Fok I cleavage domain. Proc Natl Acad Sci USA. 1996;93:1156-1160.

2. Carroll D, Charo RA. The societal opportunities and challenges of genome editing. Genome Biology. 2015;16(1):1-9.

3. Xue HY, Ji LJ, Gao AM, Liu P, He JD, Lu XJ. CRISPR-Cas9 for medical genetic screens: applications and future perspectives. J Med Genet. 2016;53:91-97.

4. Ishino $Y$, Shinagawa $H$, Makino K, Amemura M, Nakata A. Nucleotide sequence of the iap gene, responsible for alkaline phosphatase isozyme conversion in Escherichia coli, and identification of the gene product. J Bacteriol. 1987;169:5429-5433.

5. Makarova SK, Haft DH, Barrangou R. Evolution and classification of the
CRISPR-Cas systems. Nat Rev Microbiol. 2011:9:467-477.

6. Jinek M, Chylinski K, Fonfara I, Hauer M, Doudna JA, Charpentier E. A programmable dual-RNA-guided DNA endonuclease in adaptive bacterial immunity. Science. 2012;337(6096):816-821.

7. Lokody I. Correcting genetic defects with CRISPR-Cas9. Nat Rev Genet. 2014; 15:63.

8. Burgess JD. In vivo correction of genetic disease in adult mice. Nat Rev Genet. 2014; 15:291.

9. Burgess JD. Technology: A CRISPR genome-editing tool. Nat Rev Genet. 2013;14: 80-81.

10. Blake W, Esther VD, Jelle BB, el al. RNA-guided complex from a bacterial immune system enhances target recognition through seed sequence interactions. PNAS. 2011;108(25): 10092-10097.

11. Josiane EG, Marie-Eve D, Manuela V, et al. The CRISPR/Cas bacterial immune system cleaves bacteriophage and plasmid DNA. Nature. 2010;468: 67-72.

12. Davis AJ, Chen DJ. DNA double strand break repair via non-homologous end-joining. Transl Cancer Res. 2013;2(3):130-143.

13. Ledford $\mathrm{H}$. Alternative CRISPR system could improve genome editing. Nature. 2015; 526:17.

14. Bernd Z, Jonathan SG, Omar OA. Cpf1 Is a Single RNA-Guided Endonuclease of a Class 2 CRISPR-Cas System. Cell. 2015;163(3):759771.

15. Lander ES. The Heroes of CRISPR. Cell. 2016;164(1-2):18-28.

16. CRISPR-Cpf1 May Outsnip CRISPR-Cas9. GEN News Highlights. http://www.genengnews.com/gen-news-highlights/crispr-cpf1-mayoutsnip-crispr-cas9/81251791/. [ accessed 26.Feb.2015].

17. Scientists discover new system for human genome editing with potential to increase power and precision of genome engineering. Broadinstitute News. https://www.broadinstitute.org/news/7272. [accessed 23.Feb.2016].

18. Yui S, Nakamura T, Sato T, et al. Functional engraftment of colon epithelium expanded in vitro from a single adult Lgr5+ stem cell. Nat Med. 2012;18, 618-623.

19. Gerald S, Koo BK, Sasselli V. Functional Repair of CFTR by CRISPR/ Cas9 in Intestinal Stem Cell Organoids of Cystic Fibrosis Patients. Cell. 2013;13(6): 653-658.

20. Patrick DH, Eric SL. Development and Applications of CRISPR-Cas9 for Genome Engineering. Cell. 2014;157(6): 1262-1278.

21. U.S National Library of Medicine. Huntington disease. Genetic Home Reference. https://ghr.nlm.nih.gov/condition/huntington-disease. 3 May 2016. [accessed 10.04.2016].

22. U.S National Library of Medicine. HTT Huntington. Genetic Home Reference. https://ghr.nlm.nih.gov/gene/HTT. 3 May 2016. [accessed 10.04.2016].

23. Bae S, Kweon J, Kim HS, Kim JS. Microhomology-based choice of Cas9 nuclease target sites. Nat Methods. 2014;11(7):705-706.

24. Li HL, Gee P, Ishida K, Hotta A. Efficient genomic correction methods in human iPS cells using CRISPR-Cas9 system. Methods. 2015;101:2735.

25. Rajat M, Kiran M. Expanding the genetic editing tool kit: ZNFs, TALENs and CRISPR-Cas 9. The Journal of Clinical Investigation. 2014;124(10):4154-4161.

26. Sara R, Federica U, Melanie H,et al. CDKL5 ensures excitatory synapse stability by reinforcing NGL-1-PSD95 interaction in the postsynaptic compartment and is impaired in patient iPSC-derived neurons. Nat. Cell Biol. 2012;14: 911-923.

27. Brennand $\mathrm{KJ}$, Simone A, Jou J et al. Modelling schizophrenia using human induced pluripotent stem cells. Nature 2012;473: 221-225.

28. Kannan R, Ventura A. The CRISPR revolution and its impact on cancer research. Swiss Med Wkly. 2015;145:w14230.

29. Wen WS, Yuan ZM, Ma SJ, Xu J, Yuan DT. Crispr-cas9 systems: versatile cancer modelling platforms and promising therapeutic strategies. Int $J$ Cancer. 2016;138:6;1328-1336.

30. Randall J, Sidi C, Yang Z, et al. CRISPR-Cas9 knockin mice for genome editing and cancer modeling. Cell 2014;159;440-455.

31. Hu Z, Yu L, Zhu D, et al. Disruption of HPV16-E7 by CRISPR/Cas System Induces Apoptosis and Growth Inhibition in HPV16 Positive Human Cervical Cancer Cells. Biomed Res Int. 2014; Article ID:612823.

32. Tang L, Jacson KS, Zhihong L, Edwin C, Francis JH, Zhenfeng D. Development and potential applications of CRISPR-Cas9 genome editing technology in sarcoma. Cancer Letters. 2016;373(1):109-118.

33. Wen WS, Yuan ZY, Ma SJ, Xu J, Yuan DT. CRISPR-Cas9 systems: versatile cancer modelling platforms and promising therapeutic strategies. Int J Cancer. 2016;138(6):1328-36.

34. Zhen S, Takahashi Y, Narita S, Yang YC, Li X. Targeted delivery of 
CRISPR/Cas9 to prostate cancer by modified gRNA using a flexible aptamer-cationic liposome.Oncotarget. 2016. DOl: 10.18632/ oncotarget.14072.

35. Kim E, Hurtz C, Koehrer S, et al. Ibrutinib inhibits pre-BCR+ B-cell acute lymphoblastic leukemia progression by targeting BTK and BLK. Blood. 2016. doi.org/10.1182/blood-2016-06-722900.

36. Huibin T, Joseph BS. CRISPR/Cas-mediated genome editing to treat EGFR-mutant lung cancer: a personalized molecular surgical therapy. EMBO. 2016;8(2):83-85

37. Xun LX, Leqiang S, Teng $Y$, et al. A CRISPR/Cas9 and Cre/Lox systembased express vaccine development strategy against reemerging Pseudorabies virus. Sci Rep. 2016; 6:19176.

38. Money of the genes: CRISPR attracts a lot of investors. News \& Tips. 2016. http://allcompanies.website/2016/01/25/money-of-the-genescrispr-attracts-a-lot-of-investors/. [accesed 13.02.2016].

39. Wang G, Zhao N, Berkhout B, Das AT, et al. A Combinatorial CRISPRCas9 Attack on HIV-1 DNA Extinguishes All Infectious Provirus in Infected T Cell Cultures. Cell Rep. 2016;17(11):2819-2826.

40. Edward AP, Ryan BP, Benjamin JF, Jeffrey SG, Aijaz A, Robert GG. Future Therapy for Hepatitis B Virus: Role of Immunomodulators. Curr Hepatol Rep. 2016;15(4):237-244.

41. Zhengyan F, Botao Z, Wona D, et al. Efficient genome editing in plants using a CRISPR/Cas system. Cell Res. 2013; 23:1229-1232.

42. Mercx S, Tollet J, Magy B, Navarre C, Boutry M. Gene Inactivation by CRISPR-Cas9 in Nicotiana tabacum BY-2 Suspension Cell. Front Plant Sci. 7:40. doi: 10.3389/fpls.2016.00040 http://dx.doi.org/10.3389/ fpls.2016.00040. [accesed 01.02.2016].

43. Lombardo L, Coppola G, Zelasco S. New Technologies for Insect-Resistant and Herbicide-Tolerant Plants. Trends Biotechnol. 2016;34(1):49-57.

44. Ain QU, Chung JY, Kim JH. Current and future delivery systems for engineered nucleases: ZFN, TALEN and RGEN. J Control Release. 2015;205:120-127.
45. Bing S, Liz HM, Yi G, Ying P. The Rise of CRISPR/Cas for Genome Editing in Stem Cells. Stem Cells Int. 2016;Volume 2016:17 pages. Article ID 8140168: doi:10.1155/2016/8140168.

46. Cho S W, Kim S, Kim Y, et al. Analysis of off-target effects of CRISPR/ Cas-derived RNA-guided endonucleases and nickases. Genome Res. 2014; 24:132-141.

47. Tang L, Jacson KS, Zhihong L, Edwin C, Francis JH, Zhenfeng D. Development and potential applications of CRISPR-Cas9 genome editing technology in sarcoma. Cancer Lett. 2016;373(1):109-118.

48. Slaymaker IM, Gao L, Zetsche B, Scott DA, Yan WX, Zhang F. Rationally engineered Cas9 nucleases with improved specificity. Science. 2016;351(6268):84-88

49. Julie S, Jonathan W, David G. Opposition mounts to genetic modification of human embryos. http://mobile.reuters.com/article/healthNews/ idUSKBNOTK33F20151201. [accesed 10.02.2016].

50. Puping L, Yanwen X, Xiya Z et al. CRISPR/Cas9-mediated gene editing in human tripronuclear zygotes. Protein \& Cell. 2015; 6(5):363-372.

51. Xue W, Chen S, Yin H, et al. CRISPR-mediated direct mutation of cancer genes in the mouse liver. Nature. 2014;514:380-384.

52. Platt RJ, Chen S, Zhou Y, et al. CRISPR-Cas9 knockin mice for genome editing and cancer modeling. Cell. 2014:159(2):440-450.

53. Lu XJ, Qi X, Zheng DH, Ji LJ. Modeling cancer processes with CRISPRCas9. Trends Biotechnol. 2015;33:317-319.

54. Katerine S, Michael B, Annelien B, et al. CRISPR germline engineering the community speaks. Nature Biotechnology. 2015; 33:478-486.

55. Ewen C. UK scientists gain licence to edit genes in human embryos. Nature. 2016;530,18-19.

56. Yanfang F, Jennifer AF, Cyd K. High frequency off-target mutagenesis induced by CRISPR-Cas nucleases in human cells. Nat Biotechnol. 2013;31(9):822-826.

57. Roni A. UNESCO panel of experts calls for ban on "editing" of human DNA to avoid unethical tampering with hereditary traits. UNESCO Media Service. 10 May 2016. 